\title{
Mortalidad en el síndrome de abstinencia alcohólica
}

Santolaria F, González-Reimers E. Mortalidad en el síndrome de abstinencia alcohólica. An Med Interna (Madrid) 2006; 23: 305-306.

El consumo excesivo de alcohol representa un porcentaje importante de los ingresos en los Servicios de Medicina Interna de nuestro país, que supera el $20 \%$ y puede alcanzar hasta el 30\% (1-3). La mortalidad global de los ingresos por alcoholismo está próxima al $10 \%$ y no difiere sensiblemente de la del resto de pacientes (1). La diferencia estriba en que son muertes que ocurren a una edad menor y por tanto con una mayor pérdida de años de vida (4).

Cuando un paciente ingresa en el hospital y suprime bruscamente el consumo de alcohol puede aparecer el síndrome de abstinencia alcohólica (SAA). Este puede manifestarse inicialmente con síntomas "menores": temblor ansiedad, sudoración, palpitaciones (aparecen tras seis horas de abstinencia); a veces convulsiones por abstinencia; alucinaciones visuales; y puede progresar hasta la forma más grave, el delirium tremens, caracterizado por desorientación, alucinaciones, taquicardia, hipertensión, fiebre, agitación y sudoración. El delirium tremens se presenta en un pequeño porcentaje de alcohólicos, aproximadamente en el 5\% de los que tienen dependencia y suprimen el consumo sin tratamiento sustitutivo (5). El riesgo de delirium tremens está en relación con el consumo a diario, la historia de episodios previos de delirium tremens, la marginación social, la edad mayor de 30 años, una enfermedad médica intercurrente que motiva la abstinencia, aumento del BUN y de la frecuencia respiratoria, y disminución de la albúmina y de la presión arterial sistólica al ingreso, y un mayor tiempo transcurrido desde la última bebida. El riesgo de delirium tremens está en relación con el número de factores presentes: con 0 factores, el $9 \%$, con 1 factor, el $25 \%$ y con 2 o más factores el $54 \%$ desarrolla un delirium tremens $(6,7)$. Los episodios repetidos de SAA tienden a manifestarse en la misma forma que el episodio inicial: convulsiones en los que tuvieron convulsiones y delirium en los que tuvieron delirium (8).

La mortalidad del SAA depende de factores muy diversos como la edad; la existencia de enfermedades previas como cirrosis hepática y EPOC; la enfermedad aguda intercurrente que motiva la abstinencia: hepatitis aguda alcohólica, hemorragia digestiva, pancreatitis aguda, infecciones y traumatismos; el tipo de SAA, siendo mínima en la fase de temblor y alucinaciones, y aumentando en el delirium tremens, con la situación hiperadrenérgica asociada: taquicardia, arritmias, hipertensión, agitación, fiebre y sudoración intensa con deshidratación, hipopotasemia, hipofosfatemia e hipomagnesemia; y finalmente la mortalidad puede también deberse a los efectos secundarios del tratamiento como sedación excesiva, especialmente en el caso de problemas respiratorios asociados. Son factores de riesgo de mortalidad: la edad avanzada, hiperpirexia, enfermedad pulmonar previa y enfermedad hepática (9). Dichos factores coinciden con lo publicado en este número por Puerta Louro y cols. quien refiere aumento de mortalidad en relación con edad, bacteriemia, cirrosis e infección respiratoria. La mortalidad global de este estudio es baja, del $2,4 \%(10)$.

La mortalidad referida del SAA es muy variable y con una clara tendencia a disminuir a lo largo del tiempo. Desde cifras en torno al $40 \%$ a comienzos del siglo pasado (11), desciende al $15 \%$ en los años 50 (12), hasta la actualidad con cifras en torno al 8-6\% e incluso menores $(5,8,13,14)$. El descenso de la mortalidad puede ser atribuido, en principio, a un mejor tratamiento de los problemas médicos agudos, infecciones, deshidratación, alteraciones electrolíticas y a un adecuado tratamiento sustitutivo mediante administración de benzodiazepinas u otros sedantes. En el mejor de los casos, la mortalidad será la propia de la edad, la de las enfermedades previas, la de la enfermedad aguda que motivó el ingreso y la de sus complicaciones. Foy y cols. (1997) estudian 539 episodios de SAA con una mortalidad del $8.5 \%$, y refieren que sólo dos pacientes fallecen durante el síndrome de abstinencia, probablemente por sedación excesiva e insuficiencia respiratoria (14). También es posible que la mortalidad disminuya de forma artificial, bien por la inclusión de un mayor porcentaje de casos de SAA leve (temblor y alucinaciones auditivas de las fases iniciales) o bien porque se trate de pacientes sin comorbilidad ni enfermedad aguda intercurrente, en cuyo caso, con un tratamiento sustitutivo adecuado, la mortalidad puede descender hasta cerca del $0 \%$.

Ocasionalmente se produce una muerte brusca inesperada, a veces ya superado el SAA. Esta muerte súbita del alcohólico se ha atribuido a arritmias ventriculares graves en relación con 
el aumento de la actividad adrenérgica, miocardiopatía alcohólica o trastornos electrolíticos. Recientemente, Bär y cols. (2006) han demostrado una intensa disminución de la sensibilidad del reflejo de los barorreceptores en relación con la situación hiperadrenérgica propia del síndrome de abstinencia y que persiste, aunque atenuada, pasado el episodio. La relación entre esta alteración y el aumento de la mortalidad es inespecífica y se ha descrito en pacientes sin SAA (15). Otra posible causa de arritmias ventriculares graves es la prolongación del intervalo QT. Otero Antón y cols. (1997) describieron un aumento de dicho intervalo en casi el $50 \%$ de pacientes con SAA, que remitía días después. Se ha descrito el caso de una madre alcohólica, cuyo hijo recién nacido desarrolló una taquicardia ventricular en su tercer día de vida y un QT largo que remitió en unos días (16). Recientemente, Cuculi y cols. (2006) relacionan la prolongación del intervalo QT en pacientes con delirium tremens, o convulsiones por SAA, con la presencia de arritmias en el $10 \%$ de los pacientes (17).

Puerta Louro y cols. plantean la conveniencia de que los pacientes con SAA sean tratados en los servicios de medicina interna. Estamos completamente de acuerdo con dicha pro- puesta. El SAA debe ser considerado una urgencia médica en la que pueden presentarse problemas y complicaciones muy diversas: alteraciones del equilibrio hidroelectrolítico, neumonía, sepsis, hepatitis aguda alcohólica (con o sin cirrosis), pancreatitis aguda, hemorragia digestiva, convulsiones, traumatismo craneoencefálico, alteraciones de la conciencia y arritmias graves. El internista es el prototipo de médico capaz de enfrentarse a dicha situación.

Otra cuestión es el sistema de vigilancia que debe aplicarse en estos pacientes. Cuculi y cols. (2006) proponen que aquellos alcohólicos con SAA, con un QT superior a $500 \mathrm{~ms}$ deberían ser monitorizados en una unidad, de cuidados intensivos, o al menos de intermedios, para garantizar un tratamiento adecuado de las arritmias ventriculares.

\section{F. SANTOLARIA, E. GONZÁLEZ-REIMERS}

\author{
Servicio de Medicina Interna. Hospital Universitario de \\ Canarias. La Laguna. Tenerife
}

\section{Bibliografía}

1. Jarque A, González-Reimers E, Rodríguez-Moreno F, Santolaria, F, López-Lirola A, Ros R, et al. Prevalence and mortality of heavy drinkers in a general medical hospital unit. Alcohol Alcohol 2001; 36: 335-8.

2. Rodríguez Hernández AP, González Reimers E, Santolaria F, Batista N, Martínez Riera A, Conde A, et al. Prevalencia de alcoholismo en un servicio hospitalario de medicina interna. Canarias Médica 1990; 6: 29-31

3. Rodríguez ME, Cami J. Alcoholism among inpatients in a general hospital in Barcelona, Spain. Int J Addict 1988; 23: 29-46.

4. Bello LM, Saavedra P, Serra L. Evolución de la mortalidad y de los años de vida perdidos prematuramente relacionados con el consumo de alcohol en las Islas Canarias (1980-1998). Gac Sanit 2003; 17: 466-73.

5. Mayo-Smith MF, Beecher LH, Fischer TL, Gorelick DA, Guillaume JL, Hill A, et al. Management of Alcohol Withdrawal Delirium. Arch Intern Med 2004; 164: 1405-1412.

6. Ferguson JA, Suelzer CJ, Eckert GJ, Zhou XH, Dittus RS. Risk factors for delirium tremens development. J Gen Intern Med. 1996; 11: 410-4.

7. Cushman P Jr. Delirium tremens. Update on an old disorder. Postgrad Med 1987; 82: 117-22.

8. Essardas Daryanani H, Santolaria F, González Reimers E, Jorge JA, Batista López N, Martín F, et al. Alcoholic withdrawal syndrome and seizures. Alcohol Alcohol 1994; 29: 323-8.

9. Tavel ME, Davidson W, Batterton TD. A critical analysis of mortality associated with delirium tremens. Review of 39 fatalities in a 9-year period. Am J Med Sci 1961; 242: 18-29.

10. Puerta Louro R, Otero Antón E, Lorenzo Zúñiga V. Epidemiología del síndrome de abstinencia alcohólica. Mortalidad y factores de mal pronóstico. An Med Interna (Madrid) 2006; 23: 307-309.

11. Thompson WL. Management of alcohol withdrawal syndromes. Arch Intern Med. 1978; 138: 278-83.

12. Victor M, Adams RD. The effect of alcohol on the nervous system. Res Publ Assoc Res Nerv Ment Dis 1953; 32: 526-73.

13. Horstmann E, Conrad E, Daweke H. Severe course of delirium tremens. Results of treatment and late prognosis. Med Klin (Munich) 1989; 84: 569-73.

14. Foy A, Kay J, Taylor A. The course of alcohol withdrawal in a general hospital. QJM 1997; 90: 253-61.

15. Bär KJ, Boettger MK, Boettger S, Groteluschen M, Neubauer R, Jochum T, et al. Reduced baroreflex sensitivity in acute alcohol withdrawal syndrome and in abstained alcoholics. Drug Alcohol Depend 2006; (en prensa).

16. Otero-Antón E, González-Quintela A, Saborido J, Torre JA, Virgos A, Barrio E. Prolongation of the QTc interval during alcohol withdrawal syndrome. Acta Cardiol 1997; 52: 285-94.

17. Krasemann T. QT prolongation in the newborn and maternal alcoholism. Cardiol Young 2004 ; 14: 565-6.

18. Cuculi F, Kobza R, Ehmann T, Erne P. ECG changes amongst patients with alcohol withdrawal seizures and delirium tremens. Swiss Med Wkly 2006; 136: 223-7. 